\title{
Métodos antropológicos para estimativa de idade em cadáveres ou em restos mortais
}

\author{
Anthropological methods for age estimation in cadavers or \\ skeletal remains
}

\author{
Fernanda Sobral Scaramussa ${ }^{1}$, Ivan Dieb Miziara ${ }^{2}$, \\ Carmen Silvia M Galego Miziara ${ }^{3}$
}

DOI: http://dx.doi.org/10.11606/issn.2317-2770.v24i2p67-73

\begin{abstract}
Scaramussa FS, Miziara ID, Miziara CSMG. Métodos antropológicos para estimativa de idade em cadáveres ou em restos mortais. Saúde, Ética \& Justiça. 2019;24(2):67-73.

RESUMO: Introdução: A palavra antropologia deriva do grego e significa ciência que estuda os aspectos culturais e biológicos do ser humano. Na medicina legal, auxilia na identificação do indivíduo pela determinação do perfil biológico. Um dos aspectos mais importantes é a determinação da idade. Objetivos: Descrever os métodos mais utilizados para estimar a idade post mortem e avanços recentes. Métodos: Revisão bibliográfica entre 1920 a 2019. Resultados: Foram revisados os métodos de estimativa de idade em esqueletos e dentes. O estudo das suturas cranianas apresenta melhor precisão quando feito isoladamente. Na mandíbula, o ângulo formado é fundamental para a estimativa. Dos métodos dos ossos pélvicos, o de Suchey-Brooks é mais preciso que o de Todd e a técnica do Two Step Procedure é a de maior acurácia. Nos arcos costais, Kunos tem a melhor precisão. No contexto da "antropologia virtual", o uso da ressonância nuclear magnética é o mais apropriado. Na análise do esterno ocorreu variação de 5,88 anos e o uso do acetábulo foi o de maior precisão em estimativas que considerem intervalo de idade de dez anos. Discussão: A antropologia forense é uma área afetada, principalmente, pelas dificuldades na padronização de métodos. Apesar da descrição de vários estudos para estimar a idade, muitos são equivalentes, como o de Lovejoy e o de Suchey Brooks. Além disso, a maioria compartilha das mesmas limitações, como a dificuldade em estimar a idade acima dos 70 anos. Outra ressalva é que os métodos são específicos para a população do estudo e, quando aplicados em amostras distintas, há redução significativa da precisão: variou de 83$100 \%$ para $30-56 \%$ na análise do acetábulo. Assim, a estimativa da idade deve ser feita por associação de métodos, a fim de melhores resultados. Conclusão: Os métodos mais utilizados são: análise das suturas cranianas, ângulo da mandíbula, ossos pélvicos, arcos costais e dentes. Os métodos mais recentes são a "antropologia virtual" e o estudo do esterno.
\end{abstract}

DESCRITORES: Determinação da Idade pelo Esqueleto; Determinação da Idade pelos Dentes; Antropologia Forense; Autopsia; Cronologia.

\footnotetext{
1. Faculdade de Medicina da Universidade de São Paulo.

2. Faculdade de Medicina da Universidade de São Paulo.

3. Faculdade de Medicina do ABC.

Endereço para correspondência: nandascaramussa@gmail.com
} 


\section{INTRODUÇÃO}

A palavra antropologia tem origem etimológica grega, Anthropos - Humano, logos - estudo, cujo significado é a ciência que estuda os aspectos culturais e biológicos dos seres humanos. É, portanto, uma área da ciência que abrange vários pontos do conhecimento especializado, como $\mathrm{o}$ comportamento social, a linguagem, o grau de parentesco, a religião, a ancestralidade e, por fim, a evolução.

Beauthier et al. ${ }^{1}$ afirmam que, no domínio médico legal, tem-se na Antropologia Forense uma ferramenta fundamental para a identificação do ser humano por meio da determinação e da compreensão da morfologia do corpo humano íntegro, decomposto ou já esqueletizado, associadas aos métodos técnicos de identificação estabelecidos. Diante disso, é necessário um estudo comparativo e reconstrutivo, no qual se estima o perfil biológico: sexo, idade, estatura e ancestralidade.

Neste trabalho será a abordada a estimativa da idade, em cadáveres ou restos mortais não identificados, como um dos componentes para a completa identificação antropológica. Assim, ao avaliarmos um corpo ou restos mortais para fins de identificação, devemos responder a duas perguntas principais:

1) Que idade tinha o indivíduo quando morreu? Ou seja: sua idade cronológica e se esta medida é influenciada pela idade biológica deste mesmo indivíduo;

2) Há quanto tempo morreu? Ou seja: a cronotanatognose - baseada nos aspectos de conservação ou de destruição dos restos mortais daquele indivíduo.

Mediante o exposto anteriormente, em relação à construção do perfil biológico, nota-se que estimar a idade à morte é uma das tarefas mais importantes. Os estudiosos devem, assim, concentrar seus esforços em métodos que reduzam o intervalo entre as faixas etárias prováveis, tornando-as mais precisas.

\section{OBJETIVOS}

\section{Objetivo primário}

Descrever os métodos mais utilizados na estimativa de idade de cadáveres desconhecidos e/ou restos mortais (esqueletizados ou não), assim como, suas sensibilidades, especificidades e limitações.

\section{Objetivo secundário}

Detectar os avanços mais recentes na Antropologia Forense na estimativa da idade de cadáveres e restos mortais.

\section{MÉTODOS}

Revisão bibliográfica de artigos encontrados no Pubmed, Lilacs e sites de acervos de artigos e livros acadêmicos (google acadêmico) correspondente aos períodos de 1920 a 2019, utilizando os seguintes descritores disponíveis no DeCS: determinação da idade, antropologia forense, autopsia, idade e cronologia.

\section{RESULTADOS}

Segundo Rougé-Maillart et al. ${ }^{2}$, no contexto forense, os métodos de estimativa de idade mais utilizados podem ser divididos em dois: os dentários e os esqueléticos. Esses últimos, no entanto, dependem de fatores como o grau de preservação, a quantidade de material disponível, o número de fragmentos e o tempo decorrido após a morte, que influenciam diretamente na estimativa da idade. Ademais, a primazia do método, em combinação ou não, é de fundamental relevância a fim de que sejam os mais apropriados ao estado de conservação do material em análise, garantindo, assim, a qualidade dos resultados obtidos.

Meindl e Russell ${ }^{3}$ afirmam que a idade post mortem é um dos aspectos mais importantes e difíceis de estimar no contexto Médico Legal. Os métodos que serão discutidos neste artigo são baseados, principalmente, em mudanças morfológicas e degenerativas dos ossos e dos dentes, mais evidentes a partir da terceira década de vida.

Em 1942, Flamínio Fávero ${ }^{4}$ destaca que os elementos morfológicos são fundamentais na determinação da idade. Dentre eles, o mais importante e preciso seria o método das suturas cranianas.

França ${ }^{5}$ considera para a determinação da idade os seguintes fatores: "aparência, pele (formação de rugas), pelos, dentição e globo ocular”. No globo ocular, o elemento mais significativo para a estimativa da idade é o arco senil. No entanto, o autor destaca que essa característica é muito relativa, uma vez que está presente em $20 \%$ dos quadragenários e em $100 \%$ dos octogenários. Além dos fatores acima, o mesmo autor cita, também, "a relevância da radiografia dos ossos, do estudo das suturas cranianas e do ângulo da mandíbula na determinação da estimativa de idade".

Em tese de doutoramento, Costa ${ }^{6}$ objetivou estabelecer o grau de confiabilidade da análise das suturas cranianas na estimativa da idade a partir da observação do processo de soldadura de ossos cranianos mediante a avaliação do grau de apagamento das linhas de sutura na região coronal, sagital e lambdóide. A partir das informações obtidas, o autor conseguiu determinar que a avaliação das linhas de sutura pode contribuir significativamente para estimativa da idade e que elas fornecem (de modo complementar) indícios significativos para a determinação do gênero (fecham mais precocemente no feminino). Além disso, outra conclusão relevante deste estudo é que os dados obtidos de forma isolada tornam os resultados mais confiáveis e, 
para todas as suturas analisadas, o apagamento das linhas demarcatórias ocorreu mais precocemente no endocrânio.

Logan e Kronfeld ${ }^{7}$ foram os pioneiros no estudo da cronologia do desenvolvimento da mandíbula. Posteriormente, Prates ${ }^{8}$ aprimorou as técnicas de pesquisa previamente utilizadas, principalmente, no que diz respeito à determinação da relação entre os estágios de maturação óssea na mandíbula de indivíduos jovens. A partir desses estudos, a aferição do ângulo da mandíbula foi considerada a ferramenta mais importante para estimar a idade. Martin ${ }^{9}$ determina as angulações correspondentes às demais faixas etárias:

- $160^{\circ}$ a $170^{\circ}$ recém nascido

- $95^{\circ}$ a $100^{\circ}$ adultos

- $130^{\circ}$ a $140^{\circ}$ indivíduos idosos

Em 1985, Lovejoy et al. ${ }^{10}$ desenvolveram um método de análise a partir das mudanças encontradas na superfície auricular do ílio, que são bastante características do processo degenerativo. Neste estudo, os autores estabeleceram oito fases com um intervalo de cinco anos entre elas, por exemplo de 20 a 24 anos, de 25 a 29 anos e assim por diante. A classificação por fases ocorreu de acordo com as características observadas (faixa etária aproximada). Como fator limitante, destaca-se a sobreposição das fases, uma vez que as transformações morfológicas ocorrem de maneira dinâmica e simultânea, gerando fator de confusão de análise.

Os métodos de análise da sínfise púbica para a determinação da estimativa da idade foram sendo modificados e aperfeiçoados com o passar dos anos. Todd ${ }^{11}$ foi o primeiro a descrever as modificações degenerativas características da sínfise púbica e correlacioná-las com a estimativa de idade. Posteriormente, Brooks e Suchey ${ }^{12}$ aperfeiçoaram e substituíram a técnica desenvolvida por Todd $^{11}$. Para os autores, a sínfise púbica é considerada um local de envelhecimento esquelético bastante estabelecido, uma vez que apresenta modificações morfológicas extremamente definidas e características. Diante disso, foi possível desenvolver o método de estimativa de idade baseado em um sistema de seis fases de avaliação das alterações morfológicas da sínfise púbica, englobando as fases precoces e as tardias, assim como as alterações ósseas características de cada idade, com intervalo de nove anos entre elas.

Através de dados retrospectivos, Brown ${ }^{13}$ avaliou a precisão das principais técnicas de determinação da estimativa da idade utilizando a sínfise púbica. Como resultado, verificou que o método de Suchey e Brooks ${ }^{12}$ apresentava a classificação etária mais precisa $(97,9 \%)$, se comparada ao método de Tood $(70 \%)^{11}$.

Além dos métodos descritos acima, que se utilizam dos ossos corpo humano (esqueleto), e que são analisados, principalmente, pelos peritos médicos, há, também, na literatura, a descrição de técnicas de identificação humana e estimativa de idade através do exame dos dentes.
O método desenvolvido por Lamendin et al. ${ }^{14}$, em 1992, possui como objetivo estimar a idade em adultos após a morte através do estudo dos dentes. As variáveis de avaliação, que são a periodontose e a translucidez radicular, são associadas à mensuração do peso de três elementos: a periodontose, a translucidez e a raiz. A partir deste estudo, os autores demonstraram que a translucidez da raiz é o critério mais importante a ser considerado e que a periodontose é o único critério, estatisticamente independe da translucidez da raiz, que melhorou significativamente a precisão da determinação da estimativa da idade.

Já o método desenvolvido por Iscan et al. ${ }^{15}$ utilizase da quarta costela do indivíduo para estimar a idade. Consiste na análise das alterações morfológicas da face costal, correlacionando-as com intervalos de faixas etárias predeterminados. No entanto, existem limitações desta prática: quando desarticulada, a quarta costela dificilmente é identificada. Além disso, avaliação de apenas um parâmetro, a face costal, é inadequada, uma vez que, se a superfície for danificada, não será possível a aplicação do método.

Em 1999, Kunos et al. ${ }^{16}$, após críticas ao método de Iscan et al. ${ }^{15}$, desenvolveram uma técnica de estimar a idade a partir da primeira costela que utilizava três parâmetros de comparação: a face costal, a cabeça da costela e a faceta do tubérculo. A primeira costela foi escolhida por poder ser facilmente identificada (anatomia característica), mesmo quando desarticulada. $\mathrm{O}$ método elaborado pelos autores utilizou uma casuística de 182 indivíduos com a análise morfológica das três regiões citadas acima. Após comparação minuciosa, criou-se uma tabela correlacionando as alterações morfológicas identificadas com a idade estimada correspondente, determinando, assim, a base para uma sequência de seriação.

O TSP, em inglês, Two Step Procedure, foi um método desenvolvido com a finalidade de estreitar as estimativas de idade de adultos pós-morte baseado na associação de dois métodos confiáveis ${ }^{17}$ : o de Suckey e Brooks ${ }^{12}$ e o de Lamendin et al. ${ }^{14}$. Como o nome mesmo sugere, é composto por duas etapas, a saber:

$1^{\text {a }}$ etapa: análise da sínfise púbica pelo método Suchey e Brooks (SB).

Esta etapa apresenta duas subdivisões:

$1^{\text {a }}$ subdivisão: fases I, II e III pelo método $\mathrm{SB}$, correspondem a adultos jovens, cerca de até 40 anos. Logo, a estimativa de idade será o próprio intervalo cronológico correspondente à fase previamente determinada por SB.

$2^{\mathrm{a}}$ subdivisão: fases IV, V e VI pelo método $\mathrm{SB}$, correspondem a adultos maduros (40-60 anos). Essas fases são as que permitem a aplicação da segunda etapa do método TSP. 
$2^{\mathrm{a}}$ etapa: análise dentária pelo método Lamendin (LM).

Método aplicado sozinho com a análise de um único dente enraizado.

Em 2009 foi publicada uma revisão de literatura que apresentava como objetivo apontar o melhor método em estimar a idade para casos específicos a depender do tipo de material analisado ${ }^{18}$. Os autores chegaram à seguinte conclusão: para cadáveres frescos e em bom estado, o melhor método é o do TSP ${ }^{17}$; em materiais em estado de decomposição, a aplicação dos métodos biológicos (anatomia patológica e DNA). Para material esqueletizado, sugerem o $\mathrm{TSP}^{17}$ e o método de Lovejoy et al. ${ }^{19}$, para restos carbonizados a escolha vai se basear na quantidade de material disponível. Por fim, para partes do corpo, o fator determinante é a parte que se tem disponível, por exemplo, se for a sínfise púbica, a melhor opção é o de $\mathrm{SB}^{12}$.

Em 2015 foi realizado um estudo sobre o papel da radiologia no contexto forense ${ }^{20}$. Nesse estudo, os autores consideram a Ressonância Nuclear Magnética (RNM) o método de imagem mais apropriado para os procedimentos antropológicos, pois na RNM há pouca diferença interobservadores, além da disponibilização de imagens de alta qualidade, principalmente, da região da epífase e da metáfise. Como vantagens, a RNM se apresenta como um procedimento não invasivo e que pode ser aplicado em cadáveres sem a necessidade de preparo específico. Além disso, afirmam, a radiologia pode ser útil não somente na estimativa da idade, como, também, na caracterização do perfil biológico de identificação.

Contudo, apesar de apresentar bons resultados e vantagens, quando comparada a outros métodos, a Ressonância Nuclear Magnética ainda não pode ser utilizada como técnica única (isolada) de estimativa de idade. Outro ponto importante é que o acesso, para fins antropológicos, ainda é restrito e limitado na maioria dos serviços que a possuem.

Outros dois estudos sobre estimativa de idade após a morte em adultos serão comentados aqui: o primeiro é mais recente, de 2020 [Epub ahead of print], e o segundo é um pouco mais antigo, de 2007, mas importante devido aos resultados apresentados.

Com o propósito de desenvolver um método mais simples e objetivo para estimar a idade, Haj Salem et al. ${ }^{21}$ realizaram um estudo baseado na avaliação de radiografias das estruturas do esterno. Após preparação adequada e realização das radiografias, estabeleceu-se um escore de maturação óssea, baseado na fusão das partes do osso esterno e na degeneração e ossificação de sete cartilagens condrais. Os autores criaram quatro critérios: o primeiro, número 1 , correspondente à ausência total de ossificação e o de número 4 , à presença completa. As pontuações variavam de 8 a 32 pontos, dependendo das características encontradas após a análise. Os resultados obtidos foram: variação de apenas 5.88 anos entre a idade real e a estimada, sendo estatisticamente relevante. No entanto, como limitação ao método, destaca-se a dificuldade de estimar idade acima dos 40 anos.

Por fim, diante das limitações persistentes acima, Rissech et al. ${ }^{22}$ desenvolveram um estudo para a estimativa da idade de adultos após a morte fundamentado na análise de sete parâmetros distintos do osso acetábulo. A estimativa da idade post mortem para cada população teste foi calculada utilizando frequências baseadas em amostras de referências e na técnica de inferência bayesiana, que consiste na avaliação de hipóteses de máxima verossimilhança a partir do uso da fórmula de Bayes. As faixas etárias foram distribuídas em intervalos de 5, por exemplo de 15-19 anos; de 20-25 anos, e assim por diante. A idade estimada foi calculada isoladamente de acordo com o valor de distribuição esperado, ou seja, a referência previamente determinada pela inferência bayesiana. Os autores constataram que quando uma pessoa era analisada, na frequência de acordo com a amostra à qual pertencia, o nível de precisão e compatibilidade era alto, variando de 83 a $100 \%$, para um intervalo de 10 anos. Para a cada 5 anos, a precisão se reduzia, variando de 67 a $94 \%$.

Por fim, em relação aos métodos mais antigos, como o da análise da sínfise púbica ${ }^{12}$ e da superfície auricular do ílio ${ }^{19}$, o método do acetábulo ${ }^{22}$ apresentado por este estudo mostrou-se mais eficaz em indicar a faixa etária estimada, resultando, portanto, em maior precisão.

\section{DISCUSSÃO}

A antropologia forense é uma área científica afetada, principalmente, pelas dificuldades na padronização de métodos e procedimentos. Apesar de existirem, na literatura, vários métodos para estimativa de idade, muitos deles são equivalentes, uma vez que apresentam precisões semelhantes, como, por exemplo, o método de estimativa de idade pelo uso da superfície auricular do ílio ${ }^{19}$ e o de Suchey e Brooks ${ }^{12}$.

Além disso, segundo a revisão de literatura realizada por Cunha et al. ${ }^{18}$, a maioria deles compartilha das mesmas limitações, sendo a principal a dificuldade em estimar a idade acima dos 70 anos. Dos métodos de estimativa de idade post mortem, abordados neste artigo, apenas dois são capazes de avaliar a idade a partir da $5^{\text {a }}$ década: o de Lovejoy et al. ${ }^{19}$ e o Two Step Procedure ${ }^{17}$.

$\mathrm{O}$ método que utiliza as suturas cranianas apresenta como principal limitação a dificuldade em determinar a idade após os $50 \operatorname{anos}^{4,5}$. Apesar disso, Costa ressalta ser um método relevante, principalmente se as suturas são analisadas de maneira isolada (resultados mais precisos) ${ }^{6}$.

Com relação ao método da análise do ângulo da 
mandíbula, destaca-se a persistência de faixas etárias extensas para estimativa, uma vez que a classificação sugerida por Martin é dividida em apenas 3 fases: recémnascido, adultos e idosos ${ }^{9}$.

Sobre o estudo de Lovejoy et al. ${ }^{19}$, o fato de estudar variações que ocorrem em apenas uma única superfície (auricular do ílio) pode influenciar negativamente na aplicação do método, visto que pode ocorrer confusão na atribuição individual durante análise do material disponível.

Ainda relacionado aos ossos pélvicos, o método de Todd $^{11}$ foi pioneiro ao estudar as características degenerativas da sínfise púbica e seu método, em 1990, foi aperfeiçoado por Suchey e Brooks $(\mathrm{SB})^{12}$. Por meio de dados retrospectivos, autores demonstraram que o método de SB, com precisão de $97,9 \%$, é mais preciso do que o de Todd $(70 \%)^{13}$.

$\mathrm{Na}$ estimativa da idade por meio de elementos dentários, destaca-se o método de Lamendin et al. ${ }^{14}$, em que o uso da análise da transparência radicular provou ser o parâmetro mais importante dentre os observados. No entanto, como limitação, persiste a dificuldade em determinar a idade acima dos 50 anos.

Em relação aos métodos baseados no estudo dos arcos costais, a técnica de Kunos et al. ${ }^{16}$ mostrou-se mais completa, se comparada a de Iscan et al. ${ }^{15}$, uma vez que utiliza três parâmetros de análise da superfície costal e não apenas um, como sugerido por Iscan. Isso, portanto, leva a resultados mais fidedignos por reduzir os fatores de confusão durante o processo de observação e de aplicação do método.

O Two Step Procedure (TSP) é o método de escolha e o mais utilizado, atualmente, na medicina lega ${ }^{17}$. Como vantagens, apresenta-se como uma técnica fácil e simples de ser aplicada, além de possuir maior acurácia dos resultados, justamente pelo fato de fundamentar-se na associação de dois métodos: o de Suchey e Brooks ${ }^{12}$ e o de Lamendin et al. ${ }^{14}$.

Em relação à escolha do melhor método baseado no tipo de material disponível, Cunha e colaboradores concluíram que: para corpos frescos o melhor método é o de TSP ${ }^{17}$, em casos de material em estado de decomposição os métodos biológicos são os mais importantes e em material carbonizado a escolha do método, segundo os autores, vai depender do estado de preservação. Por fim, em relação à estimativa de idade pela análise de partes do corpo, o estudo indica que a técnica escolhida deve basear-se na estrutura e na quantidade de material disponível ${ }^{18}$.

No contexto de "antropologia virtual", destacase a Ressonância Nuclear Magnética (RNM) como a melhor ferramenta em estimar a idade post mortem por ser capaz de reproduzir imagens de alta qualidade que permitem estimar, não somente a idade do indivíduo como, também, seu perfil biológico. Além disso, é um procedimento não invasivo e que pode ser aplicado em cadáveres sem a necessidade de preparo específico. No entanto, o acesso à RNM, para fins antropológicos, ainda é restrito e limitado na maioria dos serviços que a possuem $^{20}$.

Com o propósito de desenvolver um método mais simples e objetivo para estimar a idade, realizou-se um estudo baseado na avaliação de radiográfica do esterno. Como resultados, os autores encontraram variação de apenas 5.88 anos entre a idade estimada e a real. Porém, para idades acima de 40 anos, o intervalo de idade estimada aumentou consideravelmente, sendo de até 12 anos a diferença entre elas ${ }^{21}$.

Outro ponto relevante a ser abordado nesta discussão, e que interfere diretamente nos resultados, é que os métodos desenvolvidos para estimar a idade são específicos para a população original de estudo. $\mathrm{Ou}$ seja, quando são aplicados em outras amostras ocorre redução significativa da precisão. A técnica que utiliza o acetábulo ilustra muito bem esse dado: para a população de base estudada a precisão variava de $83-100 \%$, em um intervalo de 10 anos. No entanto, ao ser aplicado em outra amostra, constatou-se, para o mesmo intervalo, redução dos valores para apenas $30-56 \%$.

Portanto, em vista do levantamento realizado no presente artigo, a determinação da estimativa da idade de restos humanos esqueletizados deve ser feita a partir da conjunção dos métodos, lançando-se mão dos mais antigos associados a outros mais modernos como, por exemplo, a utilização da RNM. Tudo isso para que os estudos e as informações se complementem possibilitando que se obtenha, ao final, resultados mais fidedignos e precisos.

\section{CONCLUSÃO}

A partir desta revisão bibliográfica, pode-se concluir que os métodos de estimativa de idade em adultos post mortem existentes e mais utilizados nos últimos anos são:

1) Avaliação das suturas cranianas;

2) Avaliação do ângulo da mandíbula;

3) Métodos que utilizam os ossos púbicos ${ }^{12,19}$;

4) Métodos relacionados aos elementos dentários $^{14}$;

5) Associação das técnicas de Suchey Brooks e de Lamendin no estudo do Two Step Procedure ${ }^{12,14,17}$;

6) Os estudos dos arcos costais, na estimativa da idade de adultos post mortem $^{15,16}$.

Em relação aos objetivos secundários desse estudo (i.e., métodos mais recentes de determinação da idade), destacam-se:

1) O uso da radiologia a partir do estudo radiológico do acetábulo ${ }^{22}$ e do esterno ${ }^{21}$; 
2) Os estudos que revelam a importância da Ressonância Nuclear Magnética nesse contexto $^{20}$.

\section{CONSIDERAÇÕES FINAIS}

De maneira geral, tanto para os vivos quanto para os mortos, a idade fisiológica pode ser bastante diferente da idade cronológica. Sabe-se que, quanto mais velha é a pessoa, maior é a discrepância entre as duas.

Após revisão bibliográfica das técnicas e dos estudos comparativos, dos métodos desenvolvidos e em aperfeiçoamento para a estimativa de idade em adultos post mortem, pode-se perceber que os métodos existentes ainda apresentam uma variação de idade ampla, o que os torna, na maioria dos casos, pouco precisos. Além disso, permanece ainda a dificuldade de estimar idade em indivíduos idosos, principalmente a partir da quinta década ${ }^{18}$.

Portanto, para que a escolha do método a ser utilizado seja adequada, parece-nos claro que os pesquisadores devem conhecer e possuir experiência com relação às técnicas disponíveis para estimar a idade (antigas e recentes). Vale ressaltar, também, que, às vezes, os melhores métodos não são aqueles com o menor erro padrão publicado, mas, sim, aqueles que foram testados mais vezes e em diferentes populações. Assim, tornam-se mais adequados à perícia e a um cenário específico.

Scaramussa FS, Miziara ID, Miziara CSMG. Anthropological methods for age estimation in cadavers or skeletal remains. Saúde, Ética \& Justiça. 2019;24(2):67-73.

\begin{abstract}
Introduction: The word anthropology derives from the Greek and means the science that studies the cultural and biological aspects of human beings. In forensic medicine, it helps identify the individual by determining the biological profile: ethnicity, sex, age, and height. Of these, one of the most important, in unidentified bodies, is the age. Objectives: To describe the most used methods to estimate post-mortem age as well as recent advances. Methods: Literature review of articles from Pubmed, Lilacs, and academic websites from 1920 to 2019. Results: The age estimation methods for skeletonized and dental elements were reviewed. Cranial sutures are more accurately evaluated in isolation. As for the mandible, the examination of the formed angle is fundamental for the estimation. Regarding the pelvic bone methods, the Suchey-Brooks method is more accurate than Todd's and the Two Step Procedure technique is the most accurate. For the coastal arches, Kunos has the best accuracy. In the context of "virtual anthropology", the nuclear magnetic resonance is the most appropriate tool. The analysis of the sternum showed a variation of only 5.88 years and the use of the acetabulum was the most accurate for a 10-year interval. Discussion: Forensic anthropology is an area affected mainly by difficulties in standardizing the methods. Despite the description of several studies that estimate age, many are equivalent, such as Lovejoy and Suchey Brooks. In addition, most share the same limitations, such as the difficulty in estimating ages over 70. Another important issue is that the methods are specific to the studied population. So, when they are applied to different samples, the accuracy is significantly reduced: from $83-100 \%$ to $30-56 \%$ (acetabulum studies). Therefore, the age estimate should be made by association of methods to obtain better results. Conclusion: The most used methods are analysis of cranial sutures, angle of the mandible, pelvic bones, rib bones and teeth. The most recent methods are "virtual anthropology" and the study of the sternum.
\end{abstract}

KEY WORDS: Age Determination by Skeleton; Age Determination by Teeth; Forensic Anthropology; Autopsy; Chronology.

\title{
REFERÊNCIAS
}

1- Beauthier JP, Lefèvre P, Orban R, Polet C, Grévin G, Quatrehomme G. Identification des personnes, anthropologie et taphonomie: L'anthropologie et la personne décédée. In: Beauthier JP, auteur. Traité de médecine légale. 2e édition. Bruxelles: De Boeck; 2011. p. 565- 627

2- Rougé-Maillart C, Vielle B, Jousset N, Chappard D, Telmon N, Cunha E. Development of a method to estimate skeletal age at death in adults using the acetabulum and the auricular surface on a Portuguese population. Forensic Sci Int. 2009;188(1-3):91-5. DOI: http://dx.doi.org/10.1016/j. forsciint.2009.03.019

3- Meindl RS, Russell KF. Recent advances in method and theory in Paleodemography. Annu Rev Anthropol. 1998;27:375-99. DOI: https://doi.org/10.1146/annurev. anthro.27.1.375

4- Fávero F. Medicina Legal. $2^{\mathrm{a}}$ ed. São Paulo: Livraria Martins Editora; 1942.

5- de França GV. Medicina Legal. $11^{\mathrm{a}}$ ed. Rio de Janeiro: Guanabara Koogan; 2017

6- Costa LRS. Estimativa da idade através da análise das suturas cranianas: contribuição para a antropologia forense [tese]. Piracicaba: Faculdade de Odontologia de Piracicaba, Universidade Estadual de Campinas; 2002.

7- Logan WHG, Kronfeld R. Development of the human jaws and surrounding structures from birth to the age of fifteen years. J Am Dent Assoc. 1933;20(3):379-427. DOI: https:// doi.org/10.14219/jada.archive.1933.0080

8- Prates NS. Crescimento facial e maturação óssea: 
(estudos em indivíduos dotados de oclusão normal) [tese]. Piracicaba: Faculdade de Odontologia de Piracicaba, Universidade Estadual de Campinas; 1976

9- Martin ES. A study of an Egyptian series of mandibles with special reference to mathematical methods of sexing. Biometrika. 1936;28(1-2):149-78. DOI: https://doi. org/10.1093/biomet/28.1-2.149

10- Lovejoy CO, Meindl RS, Mensforth RP, Barton, TJ. Multifactorial determination of skeletal age at death: a method with blind tests of its accuracy. Am J Phys Anthropol. 1985;68(1):1-14. DOI: https://doi.org/10.1002/ ajpa. 1330680102

11- Todd TW. 1920. Age change in the pubic bone. I. The white male pubis. Am J Phys Anthropol. 1920;3(3):467-70. DOI: https://doi.org/10.1002/ajpa.1330030301

12- Brooks S, Suchey JM. Skeletal age determination based on the male os pubis: a comparison of the Acsadi-Nemeskeri and Suchey-Brooks methods. Hum Evol. 1990;5:227-38. DOI: https://doi.org/10.1007/BF02437238

13- Brown CA. Uncertainty in skeletal aging: A retrospective study and test of skeletal aging methods at the Joint POW/MIA Accounting Command Central Identification Laboratory [dissertation]. Chico: California State University; 2009

14- Lamendin H, Baccino E, Humbert JF, Tavernier JC, Nossintchouk R, Zerilli A. A simple technique for age estimation in adult corpses: the two criteria dental method. J Forensic Sci. 1992;37(5):1373-9. DOI: https://doi. org/10.1520/JFS13327J.

15- Iscan MY, Loth SR, Wright R. Age estimation from the rib by phase analysis: white males. J Forensic Sci. 1984;29(4):1094-1104. DOI: https://doi.org/10.1520/ JFS11776J.

16- Kunos C, Simpson S, Russell K, Hershkovitz I. First rib metamorphosis: its possible utility for human age-at-death estimation. Am J Phys Anthropol. 1999;110(3):30323. DOI: https://doi.org/10.1002/(sici)10968644(199911)110:3<303::aid-ajpa4>3.3.co;2-f

17- Baccino E, Sinfield L, Colomb S, Baum TP Martrille, L. The two step procedure (TSP) for the determination of age at death of adult human remains in forensic cases [technical note]. Forensic Sci Int. 2014;244:247-51. DOI: https://doi. org/10.1016/j.forsciint.2014.09.005

18- Cunha E, Baccino E, Martrille L, Ramsthaler F, Prieto J, Schuliar Y, et al. The problem of aging human remains and living individuals: a review. Forensic Sci Int. 2009;193(1-3):1-13. DOI: https://doi.org/10.1016/j. forsciint.2009.09.008

19- Lovejoy CO, Meindl RS, Pryzbeck TR, Mensforth RP. Chronological metamorphosis of the auricular surface of the ilium: a new method for the determination of adult skeletal age at death. Am J Phys Anthropol. 1985;68(1):1528. DOI: https://doi.org/10.1002/ajpa.1330680103

20- Dedouit F, Saint-Martin P, Mokrane FZ, Savall F, Rousseau H, Crubézy E, et al. Virtual anthropology: useful radiological tools for age assessment in clinical forensic medicine and thanatology. Radiol med. 2015;120:874-86. DOI: https://doi.org/10.1007/s11547-015-0525-1

21- Haj Salem N, Saadi S, Jomaa SB, Othmani H, Hmida B, Elmhamdi S, et al. Age estimation at death by the study of chest plate radiographs: establishing a Tunisian male score. Int J Legal Med. 2020;134:775-82. DOI: https://doi. org/10.1007/s00414-019-02101-5 [Epub ahead of print].

22- Rissech C, Estabrook GF, Cunha E, Malgosa A. Estimation of Age-at-Death for Adult Males Using the Acetabulum, Applied to Four Western European Populations". J Forensic Sci. 2007;52(4):774-8. DOI: https://doi.org/10.1111/j.15564029.2007.00486.x 